\title{
Article
}

\section{Achieving Molecular Fluorescent Conversion from Aggregation-Caused Quenching to Aggregation-Induced Emission by Positional Isomerization}

\author{
Xinli Wang ${ }^{1, *+\dagger}+$, Xiang Lin ${ }^{2,+}{ }^{(D}$, Renfu Li ${ }^{3}$, Zexin Wang ${ }^{2}$, Wei Liu ${ }^{2}$, Liwei Chen ${ }^{2}$, Nannan Chen ${ }^{2}$, Tao Dai ${ }^{4}$, \\ Shitao Sun ${ }^{5}$, Zhenli Li ${ }^{5}$, Jinle Hao ${ }^{5}$, Bin Lin ${ }^{5, *}$ and Lijun Xie ${ }^{2, *(D)}$
}

\section{check for}

updates

Citation: Wang, X.; Lin, X.; Li, R.;

Wang, Z.; Liu, W.; Chen, L.; Chen, N.;

Dai, T.; Sun, S.; Li, Z.; et al. Achieving

Molecular Fluorescent Conversion

from Aggregation-Caused

Quenching to Aggregation-Induced

Emission by Positional Isomerization.

Molecules 2022, 27, 193. https://

doi.org/10.3390/molecules27010193

Academic Editors: Barbara Panunzi and Marinella Striccoli

Received: 8 December 2021

Accepted: 27 December 2021

Published: 29 December 2021

Publisher's Note: MDPI stays neutral with regard to jurisdictional claims in published maps and institutional affiliations.

Copyright: (C) 2021 by the authors. Licensee MDPI, Basel, Switzerland. This article is an open access article distributed under the terms and conditions of the Creative Commons Attribution (CC BY) license (https:// creativecommons.org/licenses/by/ $4.0 /)$.
1 Department of Oncology, Fujian Medical University Union Hospital, Fuzhou 350001, China

2 Fujian Provincial Key Laboratory of Screening for Novel Microbial Products, Fujian Institute of Microbiology, Fuzhou 350007, China; acomiy2333@gmail.com (X.L.); Wangzexin1221@126.com (Z.W.); xiguapi20@gmail.com (W.L.); liweichen202112@163.com (L.C.); 13110774889@163.com (N.C.)

3 CAS Key Laboratory of Design and Assembly of Functional Nanostructures, Fujian Key Laboratory of Nanomaterials, Fujian Institute of Research on the Structure of Matter, Chinese Academy of Sciences, Fuzhou 350002, China; lirenfu@fjirsm.ac.cn

4 State Key Laboratory of Structural Chemistry, Fujian Institute of Research on the Structure of Matter, Chinese Academy of Sciences, Fuzhou 350002, China; daitao@fjirsm.ac.cn

5 Department of Medicinal Chemistry, School of Pharmaceutical Engineering, Shenyang Pharmaceutical University, Shenyang 110016, China; sunshitao066@163.com (S.S.); 1765224871@163.com (Z.L.); 15333570330@163.com (J.H.)

* Correspondence: xinliwang528@outlook.com (X.W.); blin@syphu.edu.cn (B.L.); lijunxie8224@outlook.com (L.X.)

$+\quad$ These authors contributed equally to this work.

\begin{abstract}
In this work, we synthesized a pair of positional isomers by attaching a small electrondonating pyrrolidinyl group at ortho- and para-positions of a conjugated core. These isomers exhibited totally different fluorescent properties. PDB2 exhibited obvious aggregation-induced emission properties. In contrast, PDB4 showed the traditional aggregation-caused quenching effect. Their different fluorescent properties were investigated by absorption spectroscopy, fluorescence spectroscopy, density functional theory calculations and single-crystal structural analysis. These results indicated that the substituent position of the pyrrolidinyl groups affects the twisted degree of the isomers, which further induces different molecular packing modes, thus resulting in different fluorescent properties of these two isomers. This molecular design concept provided a new accurate strategy for designing new aggregation-induced emission luminogens.
\end{abstract}

Keywords: aggregation-induced emission; mechanofluorochromism; LDs Bioimaging

\section{Introduction}

Organic luminescent materials exhibiting an aggregation-induced emission (AIE) property have attracted a wide range of interest because of their promising applications in fluorescent probes, deformation detectors, security systems, and data storage [1-8]. Therefore, the design and synthesis of materials with AIE-active are of high significance. To date, most AIE-active materials were usually created by combining multiple design strategies, such as $\pi$-extended conjugated AIE backbones, the introduction of a donor-acceptor (D-A), the transformation from aggregation-caused quenching (ACQ) to AIE by regioisomerization strategy, and the fusion of AIE-active fluorophores with ACQ fluorophores [9-18]. Among them, the regioisomerization strategy is becoming one of the most popular ways to construct new aggregation-induced emission luminogens (AIEgens). Normally, this strategy can be achieved by migrating a bulky group to a different position. However, the positional impact of a small group on AIE and mechanofluorochromic (MFC) properties has rarely been further investigated $[19,20]$. 
In these previous works, the conversion from ACQ to AIE was realized due to the higher degree of distorted structure by migrating large groups to a different position, and the resulting compounds showed obvious AIE-active optical properties [21-24]. For example, in 2017 Anupama et al. investigated how triphenylamine (TPA) and tetraphenylethylene (TPE) units have different effects on their AIE and mechanofluorochromic properties at different positions of phenanthroimidazoles. In 2020, Li et al. investigated the migration of TPA from the rotor position of TBP-e-TPA to its bay position to obtain TBP-b-TPA. By changing the position of TPA, two compounds with different optical properties were obtained $[25,26]$. Therefore, we believe that studying the changes in optical properties caused by the migration of simple units can also achieve a further revelation of the relationship between the structure and AIE activity, which will also provide a guideline for the design of AIE materials' skeleton.

In this contribution, two new isomers named PDB2 and PDB4 are subtly designed and synthesized. Interestingly, PDB2 exhibits AIE properties, whereas PDB4 shows an ACQ effect. The MFC properties of the two compounds were further investigated due to their different fluorescent properties, and the results demonstrated that PDB2 with an AIE property showed a significant MFC behavior with a multi-stimulus response property, while PDB4 did not possess these properties except for solvatochromism. Furthermore, both the detailed single crystal structure analysis and density functional theory (DFT) theoretical results elucidate that the molecular structure, charge distribution, and packing mode play vital roles in the photophysical processes of two isomers, which helps us understand the influence of the different substituent positions on the AIE phenomenon. This work may provide a new platform for the future design of efficient AIE materials with multi-functional properties, such as mechanochromism, acidochromism, and LDs imaging ability.

\section{Results and Discussions}

\subsection{Photophysical Properties}

The UV/vis absorption and emission properties of PDB2 and PDB4 were measured in the dimethyl sulfoxide (DMSO) solution. As shown in Figure 1a, two main absorption bands of PDB2 and PDB4 were observed, respectively. Specifically, the strong absorption band of PDB2 was attributed to spin-allowed $\pi-\pi^{*}$ transitions of the compound, and the relatively weak absorption peaks could be assigned to an intramolecular charge transfer (ICT), which was induced by the D-A units, pyrrolidinyl as the electron donor group and cyano as the electron acceptor group. In addition, the intensity of the two absorption bands of PDB4 was opposite to that of PDB2, probably due to the solvatochromic effect [27]. Meanwhile, the emission peak of PDB4 was located at around $646 \mathrm{~nm}$, which was assigned to the ICT emission [28]. When the pyrrolidinyl group was changed from a para-position to ortho-position, the corresponding compound PDB2 exhibited a red-shift and peaked at $676 \mathrm{~nm}$ in the emission spectrum (Figure 1b). These results demonstrated that the ICT emission could be controlled well by changing the position of D-A units.

The AIE properties of PDB2 and PDB4 were further investigated by dissolving them in DMSO and then adding water as the poor solvent. As is shown in Figure $1 \mathrm{c}, \mathrm{d}$, two molecules displayed emission in DMSO due to the presence of a large $\pi$-conjugated structure. Upon enhancing $f_{\mathrm{w}}$ from 0 to $90 \%$, a significant increase in the PDB2 fluorescence intensity was observed and the maximum emission wavelength of PDB2 changed from $600 \mathrm{~nm}$ to $650 \mathrm{~nm}$ due to the ICT effect resulting from an increased solvent polarity. With a further increase of $f_{\mathrm{w}}$ to $99 \%$, the AIE effect diminished because parts of the molecules began to precipitate [29].

In contrast, the fluorescence intensity of PDB4 decreased gradually with the water fraction increasing. The distinct difference in the fluorescence intensity suggested a significant ACQ effect of PDB4 (Figure 1e,f). 
(a)

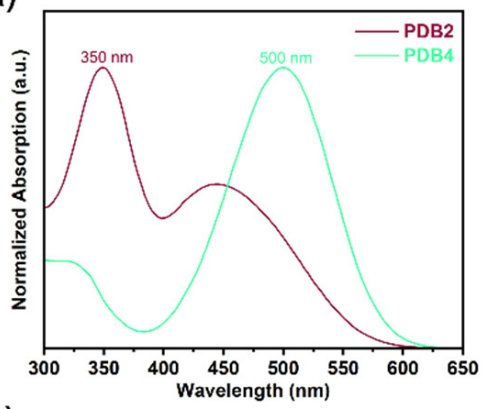

(c)

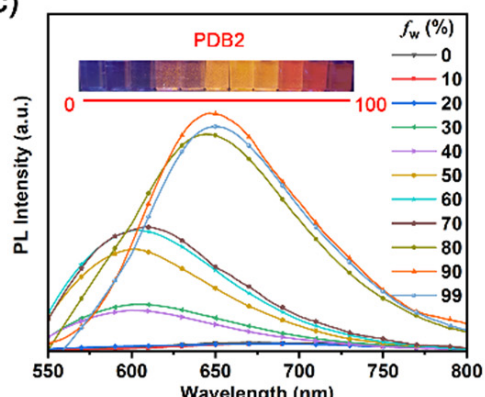

(e)

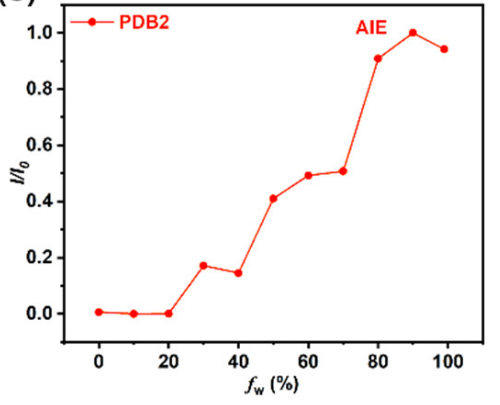

(b)

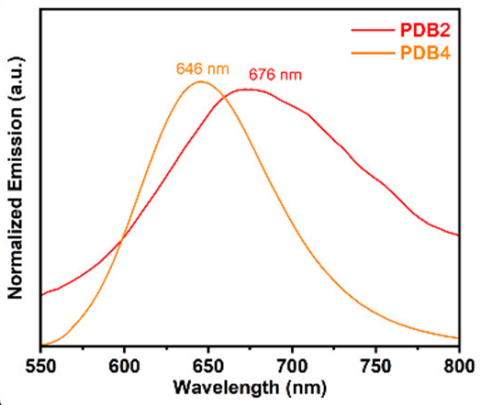

(d)

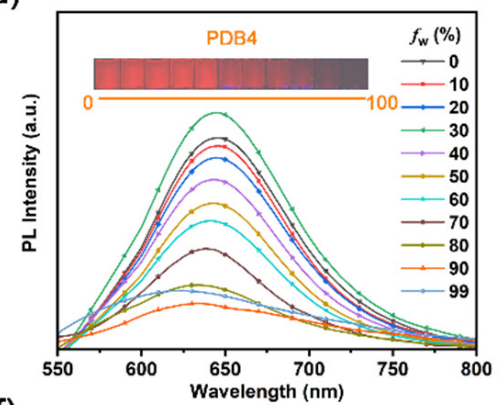

(f)

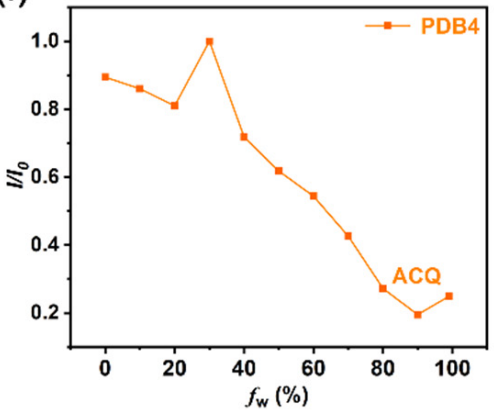

Figure 1. (a) UV/vis absorption spectra of PDB2 and PDB4 in DMSO solution (50 $\mu \mathrm{M})$. (b) PL spectra of PDB2 and PDB4 in DMSO solution $(50 \mu \mathrm{M})$. (c,d) Emission spectra of PDB2 and PDB4 in water-DMSO mixtures with different water fractions $(50 \mu \mathrm{M})$. (e) Emission intensity changes of PDB2 in different water-DMSO mixture ratios ( $\mathrm{I}_{0}$ : maximal PL intensity of $f_{\mathrm{W}}$ to 90, I: maximal PL intensity of $f_{\mathrm{W}}$ to other fraction points). (f) Emission intensity changes of PDB4 in different water-DMSO mixture ratios $\left(\mathrm{I}_{0}\right.$ : maximal PL intensity of $f_{\mathrm{W}}$ to 30 , I: maximal PL intensity of $f_{\mathrm{W}}$ to other fraction points).

\subsection{Intramolecular Charge Transfer}

To investigate the ICT effect of PDBs in different solvents with various polarities, we measured the absorption and emission spectra of PDBs in a series of solvents with different polarities. As shown in Figure S7, there was almost no significant change in the absorption spectrum with the change in solvent polarity [30]. On the contrary, by changing the solvent from weakly polar dichloromethane (DCM) to highly polar DMSO, the emission peaks of PDB2 and PDB4 underwent a red shift from 600 to $676 \mathrm{~nm}$ and from 602 to $650 \mathrm{~nm}$, respectively, which indicated a typical solvent discoloration effect (Figure 2a,b). At the same time, the fluorescent intensity of PDB2 decreased with the increase of the solvent polarity, which might be due to the positive solvatokinetic effect, while the fluorescence intensity of PDB4 increased with the increase of the solvent polarity, which might be due to the negative lysis-induced kinetic effect. This demonstrated that the ICT effect of PDB2 was stronger than that of PDB4 [29]. Meanwhile, DFT calculations on their energy levels were performed to study the ICT process of the PBDs. The Highest Occupied Molecular Orbital (HOMO) and Lowest Unoccupied Molecular Orbital (LUMO) are shown in Figure 2. The geometry in the PDB2 was more distorted than that of PDB4 (Figure 2c). The HOMO electron cloud of PDB2 was mainly located at the electron donor (i.e., pyrrole group) and 
its adjacent benzene ring, whereas the LUMO electron cloud of PDB2 was mainly located near the electron acceptor (i.e., cyan group). In contrast, the HOMO electron cloud of PDB4 was mainly delocalized over the conjugated backbone, with a small portion located at the electron donor. In contrast, the LUMO electron cloud of PDB4 was distributed similarly to that of PDB2. Meanwhile, the HOMO-LUMO gaps of PDB2 and PDB4 were $2.81 \mathrm{eV}$ and $2.72 \mathrm{eV}$, respectively, which indicated that the HOMO and LUMO of these two compounds were separated well and that PDB2 may have a stronger ICT tendency [31-33]. We also used the Lippert Mataga plot to interpret solvent-dependent spectral shifts. The Lippert Mataga plot for PDB2 and PDB4 in various solvents is shown in Figure S8 and Table S1. If DCM and ethanol are omitted, the rest of the data show a linear correlation, which suggests that the emission spectra of PDB2 and PDB4 are related to the ICT effect [34].

(a)

(c)

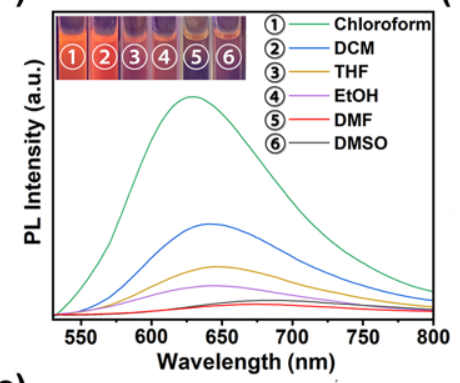

(b)
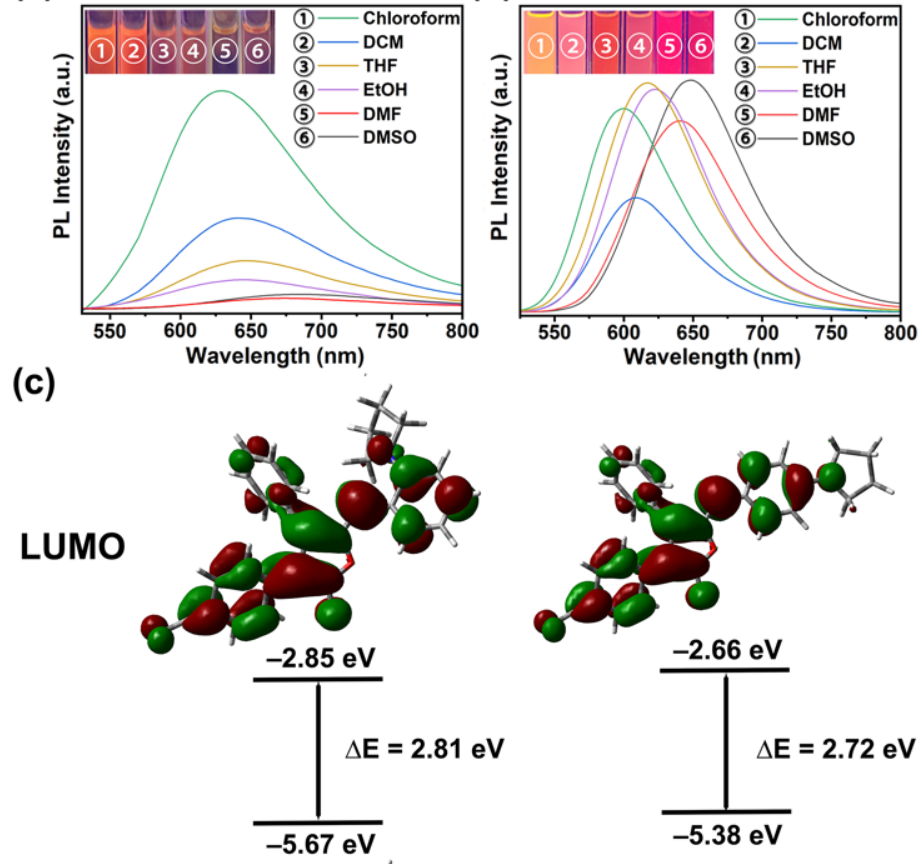

HOMO
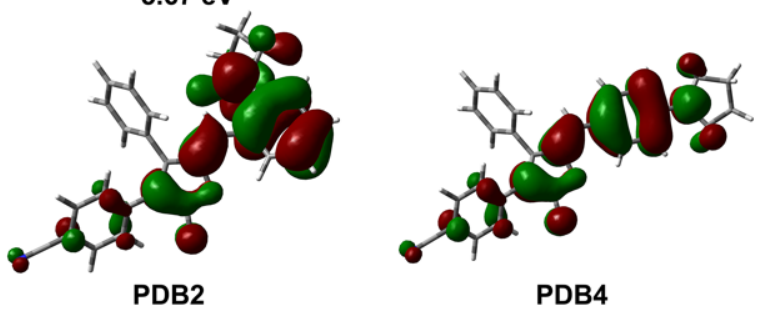

Figure 2. (a) Fluorescence spectra of PDB2 $(50 \mu \mathrm{M})$ in different solvents; (b) Fluorescence spectra of PDB4 $(50 \mu \mathrm{M})$ in different solvents. Inset: fluorescence images (Excited wavelength: $365 \mathrm{~nm}, 10 \mathrm{mM}$ ); (c) Calculation of the spatial distribution of HOMO and LUMO for PDB2 and PDB4 in DMSO.

\subsection{Crystal Structure}

The orange PDB2 crystals (CCDC 2102794) and dark red PDB4 crystals (CCDC 2102793) were obtained by slow evaporation of tetrahydrofuran and acetonitrile solution, respectively, and suitable single crystals were selected for single crystal X-ray diffraction analysis (Figure S9). The crystal data are shown in Tables S2 and S3, and the crystal structures of PDB2 and PDB4 are shown in Figure 3. It could be seen that PDB2 was packed more loosely than PDB4 in the crystal without obvious $\pi-\pi$ stacking. In PDB2, the lactone ring was packed on top of the hydrogen atom of a benzene ring, not the center of the benzene ring. Such a packing mode decreased the packing interactions significantly. In contrast, in PDB4, the two molecules were packed perfectly on top of each other between these two 
benzene rings with distance of $3.39 \AA$, and such $\pi-\pi$ interactions contributed greatly to its fluorescent properties.

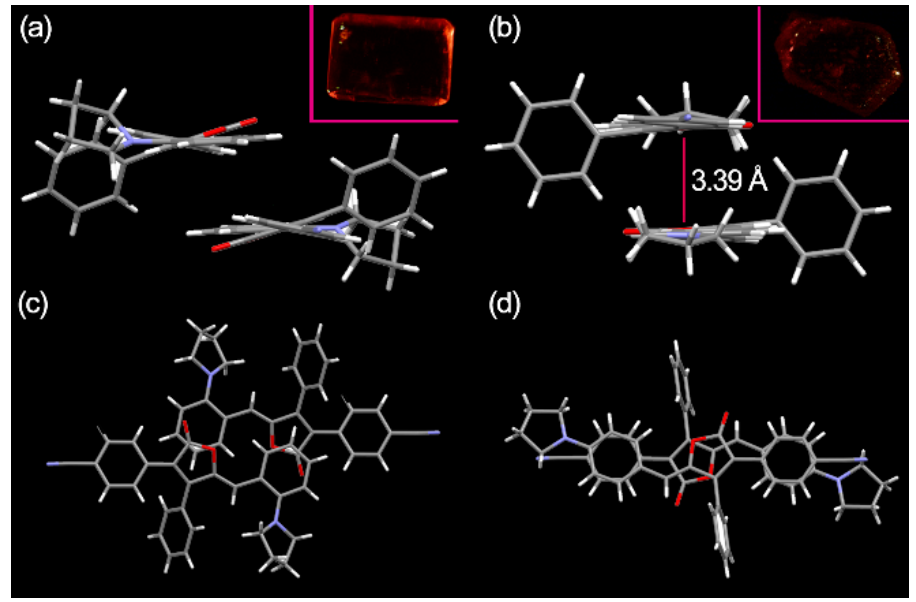

Figure 3. (a) Side view of PDB2; (b) Side view of PDB4; (c) Top view and illustration of no $\pi-\pi$ stacking in PDB2; (d) Top view and illustration of the $\pi-\pi$ stacking in PDB4. Inset: Photos of both the crystal PDB2 and PDB4 under natural light.

\subsection{Mechanofluorochromic Properties}

Recently, AIE-active materials were potential candidates for highly efficient MFC materials. Therefore, taking PDB4 as a control, we investigated the potential MFC properties of PDB2 benefiting from its good AIE properties. Under UV irradiation, the prepared powders PDB2 and PDB4 exhibited orange fluorescence and violet-red fluorescence (Figure S10) with emission peaks of $652 \mathrm{~nm}$ and $672 \mathrm{~nm}$, respectively (Figure 4a,b). After the solids of these two isomers were processed by grinding with an agate mortar, an emission redshift of about $42 \mathrm{~nm}$ to $694 \mathrm{~nm}$ was observed for PDB2. This MFC behavior of PDB2 was reversible, so that the emission color could be recovered and returned to the original emission color by heating the ground sample of PDB2 at $100{ }^{\circ} \mathrm{C}$ within 10 min or immersing it in acetone solvent. In contrast, as expected, no MFC phenomenon was observed for the corresponding PDB4. The distinctly opposite MFC phenomena based on these two isomers are worthy of attention in order to investigate the mechanism of the MFC materials.

To further explore the MFC mechanism, the PXRD patterns of the different state powders of PDB2 and PDB4 were obtained. Both pristine powders displayed intense and sharp reflection peaks. This result indicated their well-ordered crystalline nature (Figure $4 c, d$ ). On the other hand, there was a small difference in the PXRD of PDB2 in different states, and some of its diffraction curves weakened after grinding, which may be due to the red shift produced by PDB2 and attributed to planarization caused by grinding [29]. However, PDB4 was not an MFC-active material, although its physical phase transformations from a crystalline state to amorphous state happened upon grinding, as shown in Figure 4d [35].

The transition from a crystalline structure to an amorphous state when grinding PDB2 was further confirmed by DSC experiments. The DSC curves of PDB2 (Figure S11) showed an endothermic transition peak at $229^{\circ} \mathrm{C}$. For the ground sample, the endothermic transition peak changed to $228^{\circ} \mathrm{C}$. The endothermic transition peak returned to $229^{\circ} \mathrm{C}$ when the ground sample of PDB2 was heated. The endothermic transition peak of the sample immersed in acetone showed no change, but a glass transition appeared at $393.5^{\circ} \mathrm{C}$ [36-38]. However, the DSC curves of PDB4 did not exhibit properties similar to PDB2 [39]. There was no significant change in the DSC curve of PDB4. 

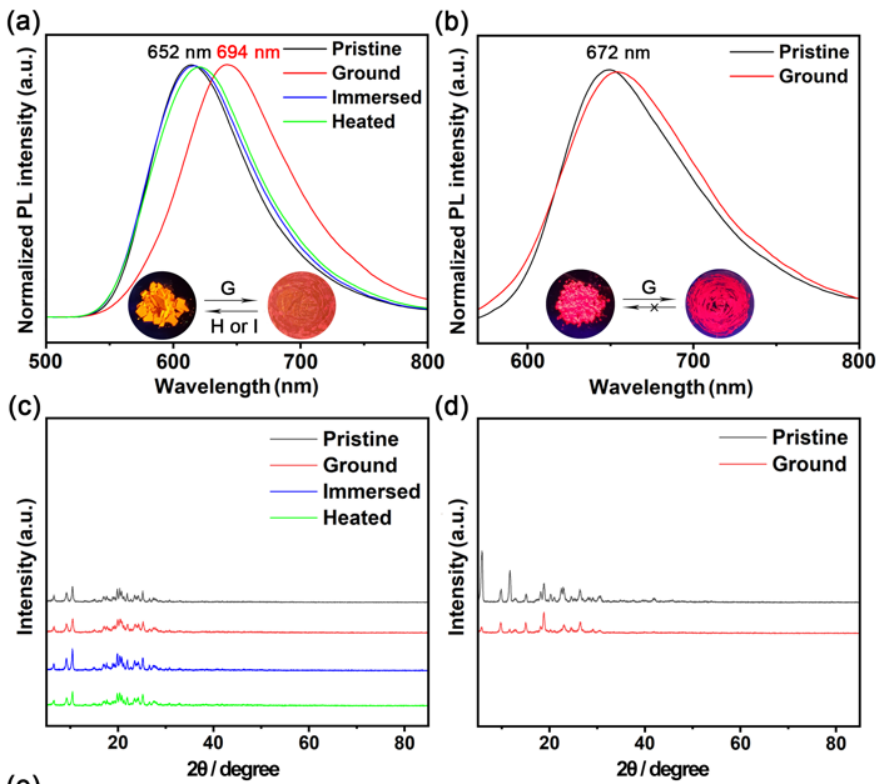

(e)
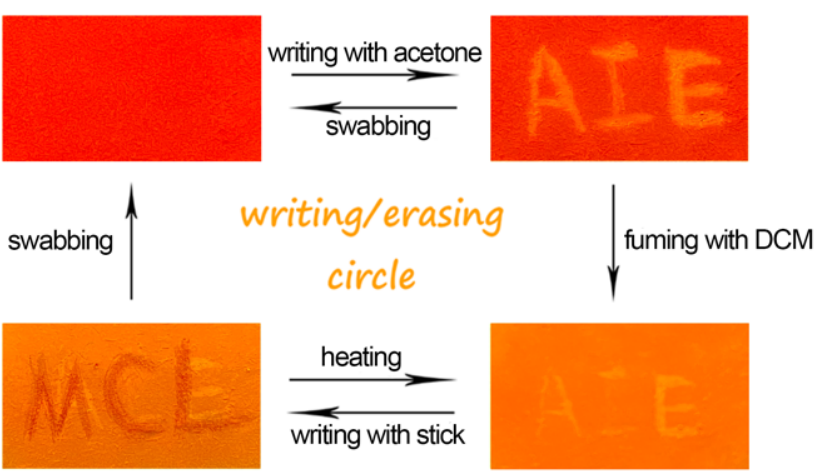

Figure 4. (a) Emission spectra of a pristine, ground sample, an immersed sample, and a heated sample of PDB2. (b) Emission spectra of a pristine, ground sample of PDB4. (c,d) Powder X-ray diffraction patterns of PDB2 and PDB4 in different states. (e) Photos of PDB2 in the writing/erasing cycle under UV light $\left(\lambda_{\mathrm{ex}}=365 \mathrm{~nm}\right)$. Inset: Photos upon irradiation with $365 \mathrm{~nm}$ UV light of PDB2 and PDB4 in different states.

Based on the mechanofluorochromic properties of PDB2, we explored a practical application in a rewritable paper. As shown in Figure 4e, the pristine powder of PDB2 was applied on a piece of weighing paper. Afterwards, the letters were written on the weighing paper with acetone, and the letters emitted yellow light under a $365 \mathrm{~nm}$ UV lamp. Then, the fluorescent letters could be easily erased by fuming with DCM for a few minutes; after that, the letters could be rewritten with a rod and wiped off again, and the writing/erasing process could be repeated many times. Therefore, this material shows great potential for applications on rewritable paper [40].

\subsection{Acidochromism}

As this mechanofluorochromic PDB2 has a basic pyrrolidinyl group, it should be acid-sensitive in a solution or solid state [41]. Therefore, we analyzed the proton-sensing ability of PDB2 as an example. When trifluoroacetic acid (TFA) of different concentrations was added, the absorption band of PDB2 in methanol $(\mathrm{MeOH})$ declined gradually around $410 \mathrm{~nm}$ and a new absorption band appeared at $350 \mathrm{~nm}$, which was enhanced when increasing the amount of TFA (Figure S12), and a new blue-shifted peak in the emission spectrum was formed (Figure 5a). 

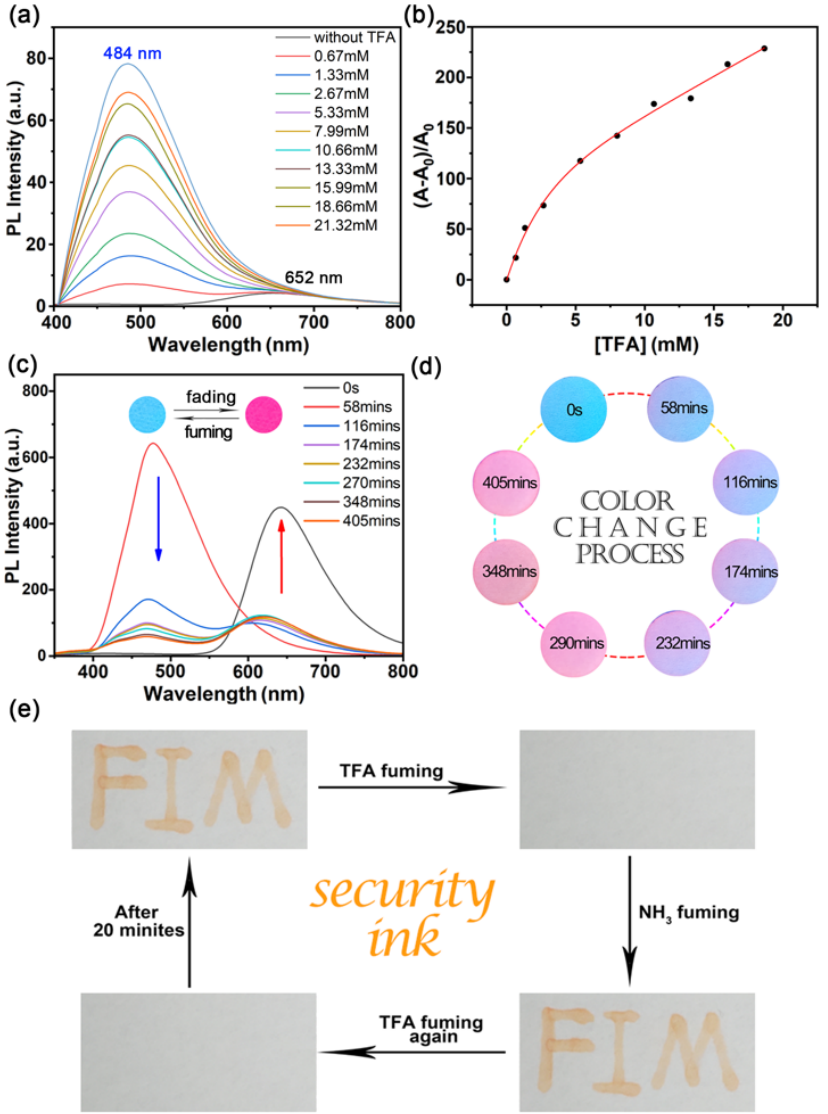

Figure 5. (a) Fluorescence spectra of PDB2 $\left(1 \times 10^{-4} \mathrm{M}\right)$ with different concentrations $(0.67-21.32 \mathrm{mM})$ of TFA in the respective solutions of $\mathrm{MeOH}$. (b) Fluorescence intensity of PDB2 at $450 \mathrm{~nm}$ versus TFA concentration. (c) Fluorescence spectra of PDB2 coated on filter paper under a gradual decrease of TFA concentration. (d) The color change process of PDB2 filter paper with a gradually decreasing TFA concentration. (e) Reversible fluorescence switching by TFA and $\mathrm{NH}_{3}$ exposure $\left(\lambda_{\mathrm{ex}}=365 \mathrm{~nm}\right)$. Inset: Photographs of circular paper sheets treated with dichloromethane PDB2 solution and circular paper sheets after TFA fuming with $365 \mathrm{~nm}$ UV light.

With the concentration of TFA increasing from 0 to $21.32 \mathrm{mM}$, the PL intensity maximum at $652 \mathrm{~nm}$ decreased and a new peak with a PL intensity maximum at $484 \mathrm{~nm}$ was formed. This change was due to the characteristic protonation of the pyrrolidine group and the formation of salt [29], while its ratio of fluorescence intensity had a quadratic function in a certain range of TFA concentrations (Figure 5b). However, the formation of this salt was not stable, and the fluorescence intensity of PDB2 was gradually recovered as time passed. Under UV light, we formed a drop of PDB2 in DCM on a piece of filter paper to form a violet-red circle, whose color changed into blue within a few seconds when fuming with TFA vapor; afterwards, the circle returned to violet-red when left at room temperature for some time. This process was further confirmed by the blue shift of the emission spectrum when treated with TFA (Figure 5c,d) [32]. In contrast, PDB4 did not react with trifluoroacetic acid (TFA) to produce these phenomena. The fluorescence of PDB4 was quenched with the addition of different concentrations of TFA, and the solution only emitted a faint red fluorescence under UV light (Figure S13) [33].

The experimental phenomenon of the acidochromic property of PDB2 inspired us to apply this compound in security inks. As depicted in Figure 5e, the letters "FIM" were written with DCM solution of PDB2 $(10 \mathrm{mM})$, and after fuming with TFA for a few seconds, the letters disappeared. Then, the letters would reappear automatically after a while. The letters would also reappear by fuming with ammonia. Moreover, after a few seconds of fuming with TFA, the letters would disappear, and they reappeared after $20 \mathrm{~min}$ [42]. 


\subsection{Lipid Drops (LDs) Staining Properties}

We further used PDB2 for the super-resolution imaging of LDs in HeLa cells to investigate the specificity of PDB2 for LDs, and co-localization experiments were performed with the lipid dye BODIPY 503. As shown in Figure 6a, HeLa cells were not stained in the bright field. The LDs in HeLa cells were detected using both PDB2 and BODIPY 503 at a concentration of $10 \mu \mathrm{M}$, respectively, to stain HeLa cells. However, at the same concentration, the staining signal-to-noise ratio of PDB2 was significantly greater than that of BODIPY 503 (Figure 6b,c). Additionally, a high overlap of the intensity profiles of PDB2 and BODIPY 503 in the region of interest (ROI) was observed, the intensity changes were closely synchronized, and the fluorescence intensity of PDB2 in the ROI was significantly greater than that of BODIPY 503 in this region (Figure 6d,e). This suggests a better selectivity of PDB2 for LDs. The staining specificity of PDB2 for LDs can be attributed to its lipophilic properties. This allows PDB2 to interact "like-like" with various neutral lipids in hydrophobic spherical LDs, allowing them to accumulate efficiently in hydrophobic spheres and producing high brightness staining properties [43,44].
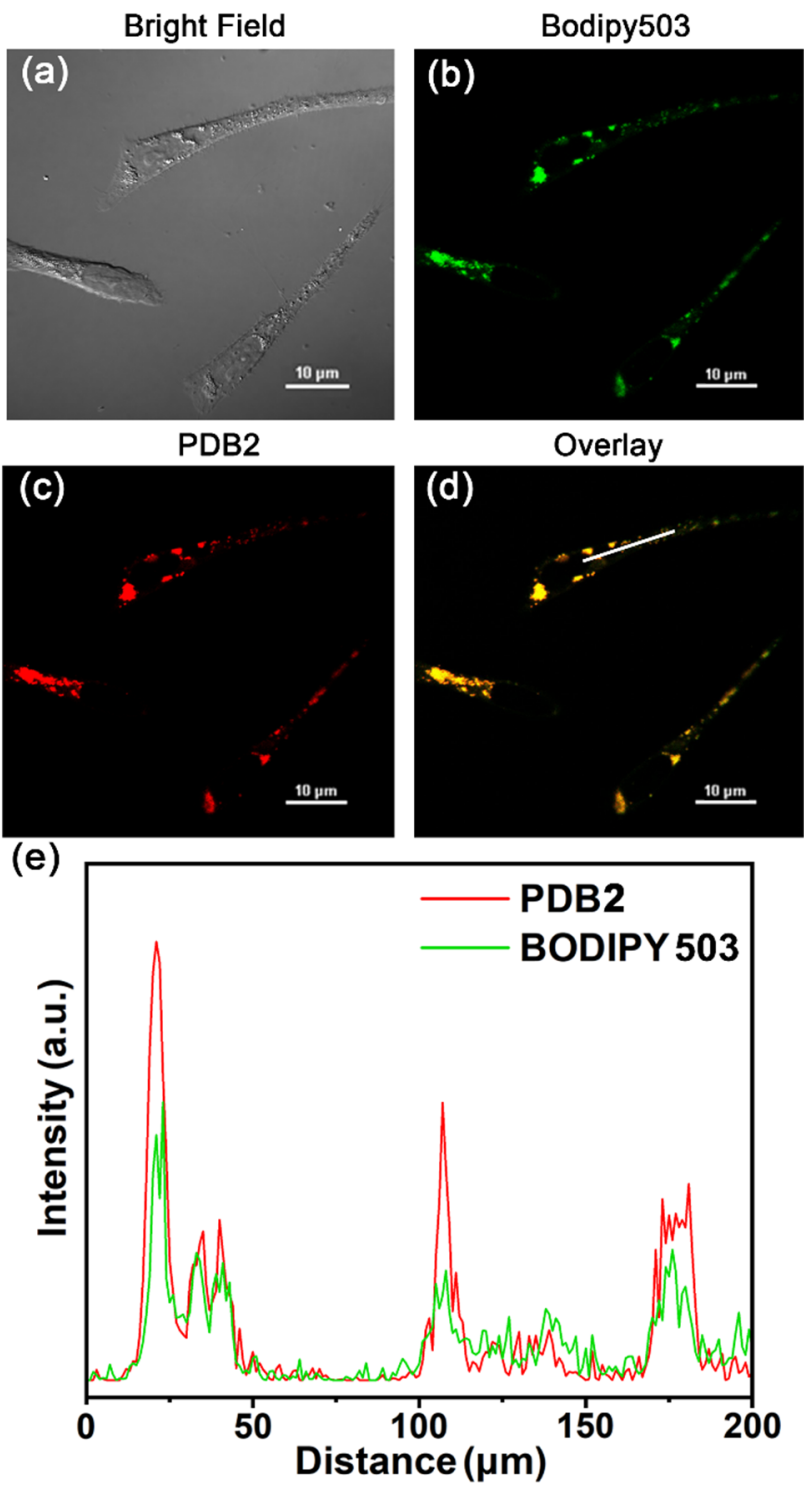

Figure 6. (a) Bright-field images of PDB2. (b,c) CLSM image of HeLa cells with BODIPY $503(10 \mu \mathrm{M})$ and PDB2 $(10 \mu \mathrm{M})$. (d) The merged images with BODIPY503 and PDB2. (e) The intensity profile of ROI lines. Scale bar $=200 \mu \mathrm{M}$ (red and green channel, excitation wavelength, etc.). 


\section{Materials and Methods}

\subsection{General Information and Materials}

All chemicals were purchased from commercial suppliers without further purification unless otherwise stated. All glassware, needles, and magnetic stirring bars were dried in a vacuum oven. Thin-layer chromatography was used to monitor reactions. ${ }^{1} \mathrm{H}$ NMR and ${ }^{13} \mathrm{C}$ NMR spectra were collected on Bruker AVANCEIII HD-400. HR-MS data were obtained using Agilent 6545 Q-TOF. Absorption and emission spectra were measured on Thermo Varioskan ${ }^{\mathrm{TM}}$ LUX Multifunctional Enzyme Labeler. The powder X-ray diffraction (PXRD) patterns were recorded on a Bruker D8 Quesr/Venture diffractometer with MoK $\alpha$ radiation $(\lambda=0.77 \AA)$. The differential scanning calorimetry (DSC) curves were determined using a heat flow DSC. The crystal structures of PDB2 and PDB4 were solved using direct methods and then refined by the full-matrix least squares refinements on F2 using the SHELXLTL software package. The absolute photoluminescence quantum yields (PLQYs) of the samples were obtained by employing a standard barium sulfate coated integrating sphere (150 mm in diameter, Edinburgh) as the sample chamber, which was mounted on the FLS980 spectrometer with the entry and output ports of the sphere located at a $90^{\circ}$ geometry from each other in the plane of the spectrometer. A standard tungsten lamp was used to correct the optical response of the instrument. All the spectral data were collected at RT and corrected for the spectral response of both the spectrometer and the integrating sphere. Lipid droplets (LDs) imaging was performed with a Nikon Ti-E\&C2 scanning unit.

\subsection{Synthesis}

The synthetic routes of PDB2 and PDB4 are shown in Scheme 1.

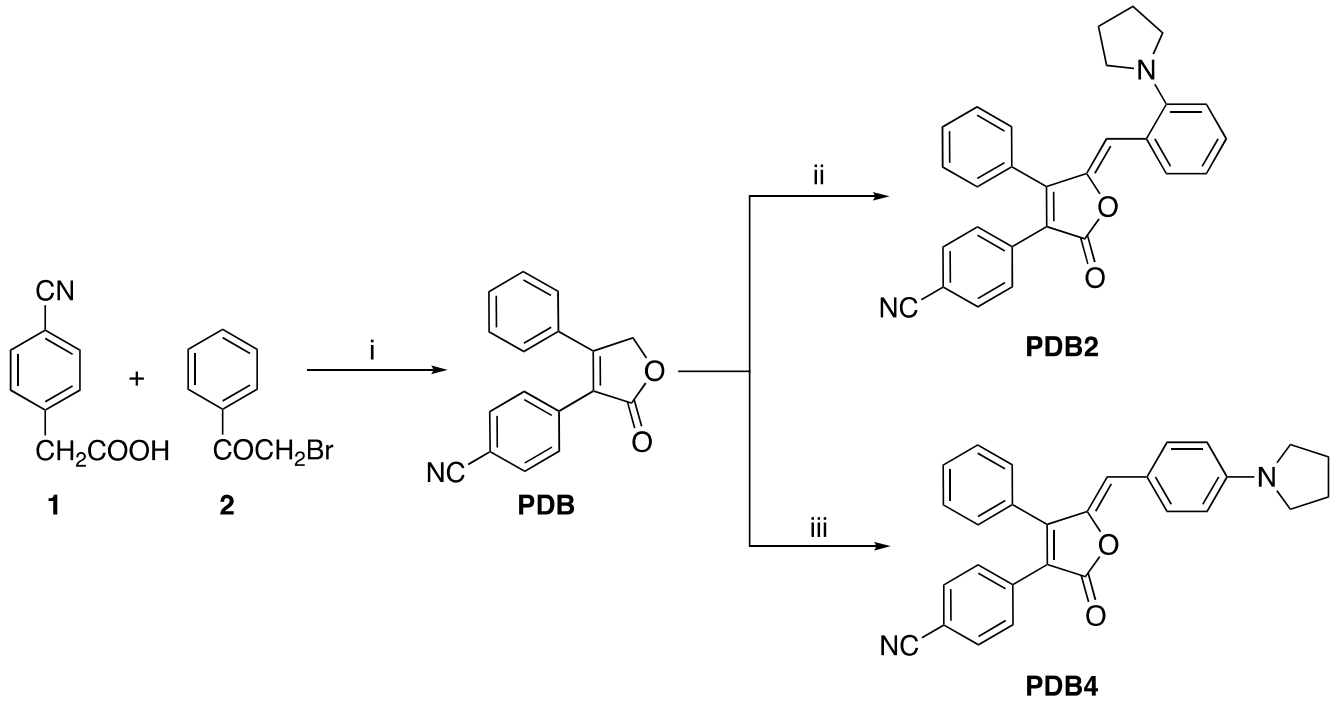

Scheme 1. Synthetic routes for PDB2 and PDB4. Reaction condition: (i) $\mathrm{Et}_{3} \mathrm{~N}, 25^{\circ} \mathrm{C}, 30 \mathrm{~min}$; DBU, $-5{ }^{\circ} \mathrm{C}, 20 \mathrm{~min}$. (ii) 2-(1-Pyrrolidinyl) benzaldehyde, piperidine, $25^{\circ} \mathrm{C}, 24 \mathrm{~h}$. (iii) 4-(1-Pyrrolidinyl) benzaldehyde, piperidine, $25^{\circ} \mathrm{C}, 24 \mathrm{~h}$.

PDB2 and PDB4 were obtained via a two-step procedure. First, 4-cyanophenylacetic acid (2.50 g, $0.015 \mathrm{~mol})$ and 2-bromoacetophenone (3.08 g, $0.015 \mathrm{~mol}$ ) were dissolved in acetonitrile solution $(65 \mathrm{~mL})$, and then triethylamine $(5.07 \mathrm{~mL})$ and DBU $(1.81 \mathrm{~mL})$ were added as catalysts to obtain PDB. Then, PDB was dissolved with two different benzaldehydes $(0.14 \mathrm{~g}, 0.798 \mathrm{mmol})$ in methanol $(10 \mathrm{~mL})$, and two drops of piperidine $(0.1 \mathrm{~mL})$ were added to obtain PDB2 and PDB4, respectively. All the compounds were characterized by ${ }^{1} \mathrm{H}$ NMR, ${ }^{13} \mathrm{C}$ NMR, HR-MS, and single crystal X-ray data. The relevant data were collected from the original spectra and are listed in the Supplementary Materials (Figures S1-S6). 


\section{Conclusions}

In summary, a new strategy for achieving the conversion from ACQ to AIE has been realized by shifting the position of a small pyrrolidinyl group on the benzene ring, which is different from traditional molecular design philosophy relying on the migration of bulky groups. This work provides new space to develop and diversify the library of AIEgens without destroying the $\pi$ conjugation system. Consequently, a new NIR AIEgens was successfully developed from these two isomers. Furthermore, the single-crystal structure analysis and DFT calculations data clearly elucidate the nature of the AIE and MFC phenomena of PDB2 at the molecular level. The AIE-active PDB2 shows a discrete cross packing mode without $\pi-\pi$ stacking, while the ACQ-dominated PDB4 adopts a long-range molecular packing mode with obvious $\pi-\pi$ stacking. Moreover, the PXRD, DSC study of these two isomers reveals that the similar physical phase transformations from a crystalline state to amorphous state were responsible for the extremely opposite MFC properties. In a further biological experiment, PDB2 shows a strong LDs imaging ability and high selectivity. This work might provide particularly valuable information for the further understanding of the AIE mechanism and supply new guidelines for the design and preparation of new promising AIEgens with multi-functional properties.

Supplementary Materials: The following supporting information can be downloaded at, the supporting information includes synthesis routes, NMR data and MS spectra, crystallographic data, and additional data of the compounds PDB2 and PDB4.

Author Contributions: Data curation, R.L., S.S., Z.L., J.H. and B.L.; Funding acquisition, X.W., R.L. and L.X.; Investigation, Z.W., W.L., L.C., N.C. and T.D.; Writing—original draft, X.L.; Writing-review \& editing, X.W. and L.X. All authors have read and agreed to the published version of the manuscript.

Funding: The Strategic Priority Research Program of the CAS: XDB20000000; Natural Science Foundation of Fujian Province: 2020J011026; Natural Science Foundation of Fujian Province: $2020 \mathrm{J06028.}$

Institutional Review Board Statement: Not applicable.

Informed Consent Statement: Not applicable.

Data Availability Statement: Not applicable.

Conflicts of Interest: The authors declare no conflict of interest.

Sample Availability: Samples of the compounds PDB2 and PDB4 are available from the authors.

\section{References}

1. Chang, Z.-F.; Jing, L.-M.; Chen, B.; Zhang, M.; Cai, X.; Liu, J.-J.; Ye, Y.-C.; Lou, X.; Zhao, Z.; Liu, B.; et al. Rational design of asymmetric red fluorescent probes for live cell imaging with high AIE effects and large two-photon absorption cross sections using tunable terminal groups. Chem. Sci. 2016, 7, 4527-4536. [CrossRef]

2. Abdurahman, A.; Wang, L.; Zhang, Z.; Feng, Y.; Zhao, Y.; Zhang, M. Novel triazole-based AIE materials: Dual-functional, highly sensitive and selective fluorescence probe. Dyes Pigm. 2020, 174, 108050. [CrossRef]

3. Kohmoto, S.; Chuko, T.; Hisamatsu, S.; Okuda, Y.; Masu, H.; Takahashi, M.; Kishikawa, K. Piezoluminescence and Liquid Crystallinity of 4,4'-(9,10-Anthracenediyl) bispyridinium Salts. Cryst. Growth Des. 2015, 15, 2723-2731. [CrossRef]

4. Zhu, X.; Liu, R.; Li, Y.; Huang, H.; Wang, Q.; Wang, D.; Zhu, X.; Liu, S.; Zhu, H. An AIE-active boron-difluoride complex: Multi-stimuli-responsive fluorescence and application in data security protection. Chem. Commun. 2014, 50, 12951-12954. [CrossRef] [PubMed]

5. $\quad$ Ravindra, M.K.; Mahadevan, K.M.; Basavaraj, R.B.; Darshan, G.P.; Sharma, S.C.; Raju, M.S.; Vijayakumar, G.R.; Manjappa, K.B.; Yang, D.-Y.; Nagabhushana, H. New design of highly sensitive AIE based fluorescent imidazole derivatives: Probing of sweat pores and anti-counterfeiting applications. Mater. Sci. Eng. C 2019, 101, 564-574. [CrossRef]

6. Pannipara, M.; Al-Sehemi, A.G.; Kalam, A.; Asiri, A.M. Naphthalene based AIE active stimuli-responsive material as rewritable media for temporary communication. Opt. Mater. 2017, 72, 442-446. [CrossRef]

7. Han, J.; Sun, J.; Li, Y.; Duan, Y.; Han, T. One-pot synthesis of a mechanochromic AIE luminogen: Implication for rewritable optical data storage. J. Mater. Chem. C 2016, 4, 9287-9293. [CrossRef]

8. Li, S.; Shang, Y.; Zhao, E.; Kwok, R.T.K.; Lam, J.W.Y.; Song, Y.; Tang, B.Z. Color-tunable and highly solid emissive AIE molecules: Synthesis, photophysics, data storage and biological application. J. Mater. Chem. C 2015, 3, 3445-3451. [CrossRef] 
9. Gao, M.; Hong, Y.; Chen, B.; Wang, Y.; Zhou, W.; Wong, W.W.H.; Zhou, J.; Smith, T.A.; Zhao, Z. AIE conjugated polyelectrolytes based on tetraphenylethene for efficient fluorescence imaging and lifetime imaging of living cells. Polym. Chem. 2017, 8, 3862-3866. [CrossRef]

10. Dai, C.; Yang, D.; Zhang, W.; Fu, X.; Chen, Q.; Zhu, C.; Cheng, Y.; Wang, L. Boron ketoiminate-based conjugated polymers with tunable AIE behaviours and their applications for cell imaging. J. Mater. Chem. B 2015, 3, 7030-7036. [CrossRef]

11. Dong, W.; Ma, Z.; Chen, P.; Duan, Q. Carbazole and tetraphenylethylene based AIE-active conjugated polymer for highly sensitive TNT detection. Mater. Lett. 2019, 236, 480-482. [CrossRef]

12. Liu, Y.; Lin, F.X.; Feng, Y.; Liu, X.; Wang, L.; Yu, Z.-Q.; Tang, B.Z. Shape-Persistent $\pi$-Conjugated Macrocycles with AggregationInduced Emission Property: Synthesis, Mechanofluorochromism, and Mercury (II) Detection. ACS Appl. Mater. Interfaces 2019, 11, 34232-34240. [CrossRef]

13. Ekbote, A.; Mobin, S.M.; Misra, R. Stimuli-responsive phenothiazine-based donor-acceptor isomers: AIE, mechanochromism and polymorphism. J. Mater. Chem. C 2020, 8, 3589-3602. [CrossRef]

14. Chua, M.H.; Zhou, H.; Lin, T.T.; Wu, J.; Xu, J. Triphenylethylenyl-based donor-acceptor-donor molecules: Studies on structural and optical properties and AIE properties for cyanide detection. J. Mater. Chem. C 2017, 5, 12194-12203. [CrossRef]

15. Qian, R.; Tong, H.; Huang, C.; Li, J.; Tang, Y.; Wang, R.; Lou, K.; Wang, W. A donor-acceptor triptycene-coumarin hybrid dye featuring a charge separated excited state and AIE properties. Org. Biomol. Chem. 2016, 14, 5007-5011. [CrossRef]

16. Guo, B.; Cai, X.; Xu, S.; Fateminia, S.M.A.; Liu, J.; Liang, J.; Feng, G.; Wu, W.; Liu, B. Decoration of porphyrin with tetraphenylethene: Converting a fluorophore with aggregation-caused quenching to aggregation-induced emission enhancement. J. Mater. Chem. B 2016, 4, 4690-4695. [CrossRef]

17. Liu, X.; Li, M.; Liu, M.; Yang, Q.; Chen, Y. From Tetraphenylfurans to Ring-Opened (Z)-1, 4-Enediones: ACQ Fluorophores versus AIEgens with Distinct Responses to Mechanical Force and Light. Chem. Eur. J. 2018, 24, 13197-13204. [CrossRef]

18. Zhao, N.; Lam, J.W.Y.; Sung, H.H.Y.; Su, H.M.; Williams, I.D.; Wong, K.S.; Tang, B.Z. Effect of the Counterion on Light Emission A Displacement Strategy to Change the Emission Behaviour from Aggregation-Caused Quenching to Aggregation-Induced Emission and to Construct Sensitive Fluorescent Sensors for $\mathrm{Hg}^{2+}$ Detection. Chem. Eur. J. 2014, 20, 133-138. [CrossRef]

19. Long, X.; Wu, J.; Yang, S.; Deng, Z.; Zheng, Y.; Zhang, W.; Jiang, X.-F.; Lu, F.; Li, M.-D.; Xu, L. Discovery of and insights into one-photon and two-photon excited ACQ-to-AIE conversion via positional isomerization. J. Mater. Chem. C 2021, 9, 11679-11689. [CrossRef]

20. Li, Y.-X.; Yang, X.-F.; Miao, J.-L.; Sun, G.-X. Substitution Position and Vinylene Bond Geometry Modulating the Fluorescence Solvatochromism and Aggregation-Induced Emission of (9-Anthryl) vinyl(1-pyrenyl) vinylbenzene Isomers. J. Phys. Chem. C 2016, 120, 21722-21729. [CrossRef]

21. Zhu, M.; Chen, Y.; Zhang, X.; Chen, M.; Guo, H.; Yang, F. Perylene bisimide with diphenylacrylonitrile on side-chain: Strongly fluorescent liquid crystal with large pseudo Stokes shift based on AIE and FRET effect. Soft Matter 2018, 14, 6737-6744. [CrossRef]

22. Zhao, Q.; Sun, J.Z. Red and near infrared emission materials with AIE characteristics. J. Mater. Chem. C 2016, 4, 10588-10609. [CrossRef]

23. Yang, X.; Zhao, Z.; Ran, H.; Zhang, J.; Chen, L.; Han, R.; Duan, X.; Sun, H.; Hu, J.-Y. New pyrene-based butterfly-shaped blue AIEgens: Synthesis, structure, aggregation-induced emission and their nondoped blue OLEDs. Dyes Pigm. 2020, 173, 107881. [CrossRef]

24. Yang, J.; Guo, Q.; Wen, X.; Gao, X.; Peng, Q.; Li, Q.; Ma, D.; Li, Z. Pyrene-based blue AIEgens: Tunable intramolecular conjugation, good hole mobility and reversible mechanochromism. J. Mater. Chem. C 2016, 4, 8506-8513. [CrossRef]

25. Ekbote, A.; Han, S.H.; Jadhav, T.; Mobin, S.M.; Lee, J.Y.; Misra, R. Stimuli responsive AIE active positional isomers of phenanthroimidazole as non-doped emitters in OLEDs. J. Mater. Chem. C 2018, 6, 2077-2087. [CrossRef]

26. Li, Y.; Liu, S.; Ni, H.; Zhang, H.; Zhang, H.; Chuah, C.; Ma, C.; Wong, K.S.; Lam, J.W.Y.; Kwok, R.T.K.; et al. ACQ-to-AIE Transformation: Tuning Molecular Packing by Regioisomerization for Two-Photon NIR Bioimaging. Angew. Chem. Int. Ed. 2020, 59, 12822-12826. [CrossRef]

27. Caruso, U.; Panunzi, B.; Diana, R.; Concilio, S.; Sessa, L.; Shikler, R.; Nabha, S.; Tuzi, A.; Piotto, S. AIE/ACQ Effects in Two DR/NIR Emitters: A Structural and DFT Comparative Analysis. Molecules 2018, 23, 1947. [CrossRef] [PubMed]

28. Ekbote, A.; Mobin, S.M.; Misra, R. Structure-property relationship in multi-stimuli responsive D-A-A' benzothiazole functionalized isomers. J. Mater. Chem. C 2018, 6, 10888-10901. [CrossRef]

29. Li, K.; Su, X.; Wang, Y.; Tao, F.; Cui, Y.; Zhang, H.; Li, T. D- $\pi$-A type barbituric derivatives: Aggregation induced emission, mechanofluorochromic and solvatochromic properties. J. Lumin. 2018, 203, 50-58. [CrossRef]

30. Tong, J.; Wang, Y.; Mei, J.; Wang, J.; Qin, A.; Sun, J.Z.; Tang, B.Z. A 1,3-Indandione-Functionalized Tetraphenylethene: AggregationInduced Emission, Solvatochromism, Mechanochromism, and Potential Application as a Multiresponsive Fluorescent Probe. Chem. Eur. J. 2014, 20, 4661-4670. [CrossRef]

31. Schmitt, V.; Moschel, S.; Detert, H. Diaryldistyrylpyrazines: Solvatochromic and Acidochromic Fluorophores. Eur. J. Org. Chem. 2013, 2013, 5655-5669. [CrossRef]

32. Chen, S.; Liu, W.; Ge, Z.; Zhang, W.; Wang, K.-P.; Hu, Z.-Q. Dimethylamine substituted bisbenzocoumarins: Solvatochromic, mechanochromic and acidochromic properties. CrystEngComm 2018, 20, 5432-5441. [CrossRef]

33. Naeem, K.C.; Subhakumari, A.; Varughese, S.; Nair, V.C. Heteroatom induced contrasting effects on the stimuli responsive properties of anthracene based donor- $\pi$-acceptor fluorophores. J. Mater. Chem. C 2015, 3, 10225-10231. [CrossRef] 
34. Subuddhi, U.; Haldar, S.; Sankararaman, S.; Mishra, A.K. Photophysical behaviour of 1-(4-N,N-dimethylaminophenylethynyl)pyrene (DMAPEPy) in homogeneous media. Photochem. Photobiol. Sci. 2006, 5, 459-466. [CrossRef] [PubMed]

35. Swathi, M.G.; Devadiga, D.; Ahipa, T.N. Mechanochromic studies of new cyanopyridone based fluorescent conjugated molecules. J. Lumin. 2020, 217, 116818. [CrossRef]

36. Gundu, S.; Kim, M.; Mergu, N.; Son, Y.-A. AIE-active and reversible mechanochromic tetraphenylethene-tetradiphenylacrylonitrile hybrid luminogens with re-writable optical data storage application. Dyes Pigm. 2017, 146, 7-13. [CrossRef]

37. Li, H.; Zhang, X.; Chi, Z.; Xu, B.; Zhou, W.; Liu, S.; Zhang, Y.; Xu, J. New Thermally Stable Piezofluorochromic AggregationInduced Emission Compounds. Org. Lett. 2011, 13, 556-559. [CrossRef] [PubMed]

38. Liu, C.; Luo, H.; Shi, G.; Yang, J.; Chi, Z.; Ma, Y. Luminescent network film deposited electrochemically from a carbazole functionalized AIE molecule and its application for OLEDs. J. Mater. Chem. C 2015, 3, 3752-3759. [CrossRef]

39. Mao, Z.; Yang, Z.; Mu, Y.; Zhang, Y.; Wang, Y.F.; Chi, Z.; Lo, C.C.; Liu, S.; Lien, A.; Xu, J. Linearly Tunable Emission Colors Obtained from a Fluorescent-Phosphorescent Dual-Emission Compound by Mechanical Stimuli. Angew. Chem. 2015, 127, 6368-6371. [CrossRef]

40. Ma, Y.; Zhang, Y.; Kong, L.; Yang, J. Mechanoresponsive Material of AIE-Active 1,4-Dihydropyrrolo [3,2-b] pyrrole Luminophores Bearing Tetraphenylethylene Group with Rewritable Data Storage. Molecules 2018, 23, 3255. [CrossRef]

41. Takahashi, S.; Nagai, S.; Asami, M.; Ito, S. Two types of two-step mechanochromic luminescence of phenanthroimidazolylbenzothiadiazoles. Mater. Adv. 2020, 1, 708-719. [CrossRef]

42. Li, Y.; Wang, X.; Zhang, L.; Liu, L.; Wang, Q.; Lu, H.; Zhao, X. Remarkable solid-state fluorescence change from the visible to the near-infrared region based on the protonation/deprotonation of an AIEgen. Mater. Chem. Front. 2020, 4, 3378-3383. [CrossRef]

43. Gao, M.; Su, H.; Li, S.; Lin, Y.; Ling, X.; Qin, A.; Tang, B.Z. An easily accessible aggregation-induced emission probe for lipid droplet-specific imaging and movement tracking. Chem. Commun. 2017, 53, 921-924. [CrossRef] [PubMed]

44. Sun, K.; Zhang, Y.-L.; Chen, X.-L.; Su, H.-F.; Peng, Q.-C.; Yu, B.; Qu, L.-B.; Li, K. A Type of Atypical AIEgen Used for OnePhoton/Two-Photon Targeted Imaging in Live Cells. ACS Appl. Bio Mater. 2020, 3, 505-511. [CrossRef] 